\title{
The Role of Dynamic Magnetic Resonance Imaging in Cervical Spondylotic Myelopathy
}

\author{
John Paul Kolcun, Lee Onn Chieng, Karthik Madhavan, Michael Y. Wang \\ Department of Neurosurgery and Miami Project to Cure Paralysis, University of Miami Miller School of Medicine, Miami, FL, USA
}

Dynamic spinal cord compression has been investigated for several years, but until the advent of open MRI, the use of dynamic MRI (dMRI) did not gain popularity. Several publications have shown that cervical cord compression is both static and dynamic. On many occasions the evaluation of cervical spondylotic myelopathy (CSM) is straightforward, but patients are frequently encountered with a significantly worse clinical examination than would be suggested by radiological images. In this paper, we present an extensive review of the literature in order to describe the importance of dMRI in various settings and applications. A detailed literature review was performed in the Medline and Pubmed databases using the terms "cervical spondylotic myelopathy", "dynamic MRl", "kinetic MRI", and "myelomalalcia" for the period of 1980-2016. The study was limited to English language, human subjects, case series, retrospective studies, prospective reports, and clinical trials. Reviews, case reports, cadaveric studies, editorials, and commentaries were excluded. The literature search yielded 180 papers, 19 of which met inclusion criteria. However, each paper had evaluated results and outcomes in different ways. It was not possible to compile them for meta-analysis or pooled data evaluation. Instead, we evaluated individual studies and present them for discussion. We describe a number of parameters evaluated in 2661 total patients, including dynamic changes to spinal cord and canal dimensions, transient compression of the cord with changes in position, and the effects of position on the intervertebral disc. dMRI is a useful tool for understanding the development of CSM. It has found several applications in the diagnosis and preoperative evaluation of many patients, as well as certain congenital dysplasias and Hirayama disease. It is useful in correlating symptoms with the dynamic changes only noted on dMRI, and has reduced the incidence of misdiagnosis of myelopathy.

Keywords: Cervical spondylotic myleopathy; Dynamic imaging; Magnetic resonance

\section{Introduction}

Cervical spondylotic myelopathy (CSM) is a disease of the elderly ( $>55$ years old), with significant stenosis seen in $90 \%$ of the population by the seventh decade [1-6]. With age, gradual degenerative changes are seen in the spine. However, there is a very poor correlation between the degree of radiological compression and the symptoms experienced by individuals. As originally suggested by
Matsunaga [7], the compression of the cervical cord with resultant myelopathy occurs due to the hypertrophy of adjacent ligaments and the formation of osseous bony spurs, which gradually compress the spinal cord while in static positions. This can be exaggerated with flexion and extension motions, as the cord is stretched and draped over the bony spondylosis. These additional stressors may add to the repetitive insults suffered by the already compressed cord, which has minimal space for compensatory

Received Jan 10, 2017; Revised Apr 11, 2017; Accepted Apr 18, 2017

Corresponding author: Lee Onn Chieng

University of Miami School of Medicine, 1600 NW 10th Ave \#1140, Miami, FL 33136, Miami, Florida 33136-1015, USA

Tel: +1-319-356-1616, E-mail: cxl383@med.miami.edu 
displacement. This trauma accumulates over time, leading to myelomalacia of the cord and irreversible deleterious changes. The myelomalacia is paradoxically visualized as an ample subarachnoid space surrounding the cord.

With the advent of open configuration MRI systems, dynamic or kinematic imaging has become possible. This allows further evaluation of physiological and pathological changes in the relationships of the intervertebral disc, the spinal canal, and the neural foramina, as well as the assessment of segmental instability in physiologic body positions. Dynamic MRI (dMRI) includes separate scans taken with the neck in flexion and extension, and could more accurately identify sites of pathologic stenosis or compression among patients with cervical spondylotic disease. Unlike myelography, such a non-invasive tool allows spine surgeons to assess the dynamic alteration of the spinal canal in vivo.

In this review, we evaluate manuscripts from the literature and summarize the importance of dynamic studies in the diagnosis of CSM.

\section{Methods}

We performed a detailed literature search in the Medline and PubMed using the key terms "cervical spondylotic myelopathy", "dynamic MRI", "kinetic MRI", and "myelomalalcia”. All studies from 1913 to 2016 were reviewed. The study was limited to English language. Studies were limited to human subjects, with the exception of one study performed on dogs which was included for historical significance. We included all age groups in our search, but most studies predominately dealt with an elderly cohort. Case series, retrospective studies, prospective reports, and clinical trials which reported flap usage following spine surgery were included. Reviews, case report, cadaveric studies, editorials, and commentaries were excluded.

\section{Results}

Our search returned 19 articles which met criteria for inclusion in this review [8-26], 7 of these were retrospective analyses; the remainder were prospective studies. 2661

Table 1. Studies included for review: demographics

\begin{tabular}{|c|c|c|c|}
\hline Study & No. of patient & Sex ratio (male:female) & Age, mean (range) \\
\hline Dalbayrak et al., 2013 [8] & 258 & 135:123 & $51(32-73)$ \\
\hline Endo et al., 2014 [9] & 62 & 28:34 & 42.1 (NR) \\
\hline Fei et al., 2010 [10] & 407 & $174: 233$ & 44.1 (19-93) \\
\hline Fujimori et al., 2013 [11] & 60 & $40: 20$ & $63.1(52.74)$ \\
\hline Hayashi et al., 2014 [12] & 435 & $214: 221$ & $49.8(20-79)$ \\
\hline Kim et al., 2014 [13] & 32 & $20: 12$ & $60.1(\mathrm{NR})$ \\
\hline Liu et al., 2015 [14] & 110 & $46: 64$ & 56.9 (NR) \\
\hline Machino et al., 2012 [15] & 527 & $331: 189$ & $62.2(23-93)$ \\
\hline Maruo et al., 2014 [16] & 45 & $33: 12$ & $66.9(50-85)$ \\
\hline Miura et al., 2009 [17] & 40 & $24: 16$ & 45.6 (NR) \\
\hline Miyata et al., 2006 [18] & 60 & $34: 26$ & $68(47-81)$ \\
\hline Muhle et al., 1998 [19] & 81 & $46: 35$ & $58(30-74)$ \\
\hline Muhle et al., 2001 [20] & 30 & $15: 15$ & $25(22-32)$ \\
\hline Muhle et al., 1998 [21] & 46 & $24: 22$ & $51(24-84)$ \\
\hline Muhle et al., 1998 [22] & 40 & $20: 20$ & $33(24-64)$ \\
\hline Seki et al., 2015 [23] & 121 & NR & NR \\
\hline White et al., 2007 [24] & 206 & $69: 137$ & 48 (15-93) \\
\hline Zeitoun et al., 2015 [25] & 51 & $33: 18$ & $62.6(36-89)$ \\
\hline Zhang et al., 2011 [26] & 50 & $31: 19$ & $60.3(38-90)$ \\
\hline Totals & 2,540 & $1,317: 1,216$ & $52.9(15-93)$ \\
\hline
\end{tabular}

NR, not reported. 
total patients were included in this review, with an average age of 52.9 years (range, $15-93$ years); $49.5 \%$ of patients were male (Table 1$)$.

Several studies included evaluated changes in spinal cord or canal dimensions during flexion/extension. While the degree of flexion/extension leading to canal compromise was quite obvious, many papers suggested greater canal compromise in the extension position. In the presence of significant anterior compression (e.g., disc bulge) the compression worsened with flexion. Poor outcomes were noted in patients with compression present even on flexion. We also reviewed various applications of dMRI in congenital skeletal dysplasia, Hirayama disease, rheumatoid disease, and achondroplasia (Table 2).

\section{Discussion}

Olsson [27], utilizing a dog model, first described the impact of dynamic compression in a 1958 study. He noted that dynamic changes in the spine sustain negative symptoms, and that removing these factors leads to reduced inflammation and improved outcomes in several compression models $[27,28]$. Recently, dMRI has gained popularity with the introduction of open and standing MRI machines, and is useful when there is a discrepancy between a poor clinical examination and radiological abnormality of myelomalacia observed in certain patients [12]. It has also been applied in younger population with symptoms of myelopathy [29]. Spinal elements undergo several changes during dMRI that are not appreciated in neutral images. In flexion, the cord is strained as the anterior osteophytic components are pronounced with retrobulging of the disc. While in extension, the cord shortens and thickens, which leads to compression by posterior elements as the canal narrows [8,9]. In many patients, a high intramedullary signal is only appreciated in dynamic positions [26]. This was further confirmed by somatosensory evoked potentials (SSEP) performed during the MRI, which showed decreased N20 amplitude in extension [30]. The neutral MRI is unable to detect the severity of compression in flexion/extension positions in patients with moderate stenosis, which often leads to delayed treatment

Table 2. Studies included for review: parameters

\begin{tabular}{|c|c|c|c|}
\hline Study & Design & Patient type & Study parameters \\
\hline Dalbayrak et al., 2013 [8] & Prospective & CSM & Canal diameter \\
\hline Endo et al., 2014 [9] & Prospective & Healthy & Cord/canal length \\
\hline Fei et al., 2010 [10] & Prospective & Disc herniation & Translational/angular motion, disc height \\
\hline Fujimori et al., 2013 [11] & Retrospective & CSM, OPLL & Cobb angles, range of motion \\
\hline Hayashi et al., 2014 [12] & Retrospective & CSM & Listhesis, compression, disc changes \\
\hline Kim et al., 2014 [13] & Retrospective & 22 CSM, 10 healthy & Compression levels \\
\hline Liu et al., 2015 [14] & Retrospective & CSM & Clinical outcomes \\
\hline Machino et al., 2012 [15] & Prospective & CSM & Cobb angles, range of motion \\
\hline Maruo et al., 2014 [16] & Retrospective & CSM, OPLL & Clinical outcomes \\
\hline Miura et al., 2009 [17] & Prospective & 20 CSM, 20 healthy & Canal/cord diameter (L-value) \\
\hline Miyata et al., 2006 [18] & Prospective & 40 CSM, 20 healthy & Canal/cord diameter (L-value) \\
\hline Muhle et al., 1998 [19] & Prospective & Healthy & Listhesis, canal/cord diameter \\
\hline Muhle et al., 2001 [20] & Prospective & CSM & Stenosis, cord compression \\
\hline Muhle et al., 1998 [21] & Prospective & CSM & Stenosis, cord impingement \\
\hline Muhle et al., 1998 [22] & Prospective & Healthy & Neuroforamen compression \\
\hline Seki et al., 2015 [23] & Retrospective & CSM & Clinical outcomes \\
\hline White et al., 2007 [24] & Retrospective & Cervical sx & Listhesis \\
\hline Zeitoun et al., 2015 [25] & Retrospective & CSM & Cord compression, HIL \\
\hline Zhang et al., 2011 [26] & Prospective & CSM & Cord/canal dimensions/impingement, HIL \\
\hline
\end{tabular}

CSM, cervical spondylotic myelopathy; OPLL, ossification of the posterior longitudinal ligament; sx, symptoms; HIL, hyperintensed intramedullary lesion. 
of the underlying pathology. We believe this is likely one of the reason for the rapid deterioration of clinical symptoms often noted in elderly populations [31].

\section{Brief description dMRI studies}

Patients typically examined at $1.5 \mathrm{~T}$ MRI with special functional positioning device. Despite there are various manufacturers in the current market, most devices are essentially pneumatically driven and mechanically allowing dynamic examination of the spine from the MRI control room. All devices allow flexion and extension while a few sophisticated devices allow lateral bending or rotation. The ideal angles used by most studies for flexion and extension were $40-45$. However patients were allowed to stop moving if they experienced pain or neurological deficits.

\section{Canal dimensions}

Muhle et al. [19] used dMRI to evaluate 81 patients aged 30-74 years with different stages of cervical spine degeneration. They observed that patients with complete obliteration of the subarachnoid space and those with severe canal compromise had significant worsening of the canal diameter during flexion/extension. They also observed worsening compression during flexion in patients with multiple disc herniations, caused by a cord draping effect. In patients with very severe stenosis, the motion was quite limited due to facet and ligament hypertrophy. These patients had worsening of the canal diameter in extension.

Utilizing these data, a classification system of 5 stages was proposed to describe the degree of pathology in patients with CSM. In Stage 0, the cervical spine is normally aligned. In Stage I, disc pathology is present (herniation, protrusion), but the cord is unaffected and any narrowing of the canal on flexion/extension does not impinge the cord. In Stage II, cervical spondylosis is present, and the cord is impinged anteriorly on flexion/extension. In Stage III, cervical spondylosis is present with restricted motion, and anterior or posterior impingement can be present. Finally, in Stage IV, CSM is present and impingement can occur anteriorly, posteriorly, or both, at one or multiple levels (Table 3).

In another study, Muhle et al. [22] evaluated the subarachnoid spaces in the cervical spine of healthy individuals. They noted that during flexion there was narrowing
Table 3. Cervical spondylotic myelopathy (CMS) classification (Muhle et al., 1998 [19])

\begin{tabular}{|c|c|}
\hline Stage & Features \\
\hline 0 & Physiologic alignment \\
\hline 1 & Disc pathology, canal narrowing, no cord impingment \\
\hline$\|$ & Spondylosis, anterior cord impingement \\
\hline III & $\begin{array}{l}\text { Spondylosis, restricted motion, anterior or posterior im- } \\
\text { pingement }\end{array}$ \\
\hline IV & CSM, anterior and/or posterior impingement \\
\hline
\end{tabular}

of $43 \%$ of the ventral space and widening of $83 \%$ of the dorsal space as compared to neutral position. During extension, the dorsal space was reduced by $17 \%$ whereas the ventral space increased by $9 \%$. In elderly populations, there is compromise in the canal space already due to degeneration of the disc, which leads to anterior cord impingement and hypertrophy of the ligamentum flavum, which reduces the global canal diameter. prolonged period of compression compromises oxygen and nutrition delivery due to microvasculature obliteration, which can also cause myelomalacia. This phenomenon accelerates in patients with congenital stenosis of the spine, dystonia, achondroplasia, and athetoid movement disorder.

A third study by Muhle et al. [21] prospectively examined stenotic changes in 46 symptomatic CSM patients with flexion/extension during dMRI. Stenosis was significantly greater in extension as compared to flexion positions, although cord compression was diagnosed in both positions. Patients with degenerative changes at multiple segments were more likely to experience dynamic compression.

Dalbayrak et al. [8] described changes in several dimensions of the spinal canal during flexion and extension. In addition to demonstrating the superior accuracy of MRI compared to CT or X-ray, they report dynamic changes in canal diameter, taking measurements in the sagittal plane. On average, the canal underwent a $14.9 \%$ expansion during flexion and a $13.4 \%$ reduction in diameter during extension. Additionally, dMRI was superior to X-ray at detecting unstable levels: whereas X-ray detected instability in $25.6 \%$ of cases, dMRI identified affected levels in $54.3 \%$.

Zeitoun et al. [25] report changes in cervical canal stenosis at multiple levels in 51 consecutive patients. A total of 255 levels were examined at rest, flexion, and extension. Utilizing Muhle's classification system (Table 3), they 
noted increased staging at all levels during extension, as compared to neutral or flexed positions. In the mid-low cervical spine (C3-6), almost one quarter of levels which were stage 1 at rest were classified as stage 3 on extension (22.5\%). These changes in canal diameter were largely under-detected by neutral-position imaging.

Kim et al. [13] investigated the role of extension MRI in determining cervical compression levels. They identified a significantly higher number of compression levels in cervical degenerative myelopathy patients (91\%) as compared to the non-myelopathy group (30\%). Elderly age was shown to predict increased stenosis in extension. With utilization of dynamic scans, clinician agreement and consensus in interpretation improved.

White et al. [24] retrospectively analyzed changes in spondylolisthesis on flexion/extension images in 258 symptomatic patients. Of 23 patients with listhesis on neutral imaging, 6 of these had changes of 2-4 mm between flexion and extension. Two patients had a listhesis during flexion/extension that was undetected on neutral images.

\section{Cord dimensions}

Endo et al. [9] describe dynamic changes in cord length in 62 patients with symptomatic CSM utilizing dMRI. They found that cord length was greatest during flexion, and shortest in extension, and the dimensions of the spinal canal changed with the cord. Cord elongation was greatest posteriorly, at the site of the posterior canal, and shortest anteriorly.

Zhang et al. [26] examined cord length and sagittal diameter during flexion/extension in 50 symptomatic CSM patients, as well as the amount of available space for the cord in the canal, degree of stenosis and cord impingment, and high-intensity intramedullary signal changes. They found that cord length was greatest in flexion and shortest in extension. In contrast to Endo et al., they found that cord length was greatest in the anterior aspect, regardless of position. Cord sagittal diameter was greatest in extension between $\mathrm{C} 4-\mathrm{C} 7$. In all levels but $\mathrm{C} 6$, the available space for the cord was greatest in neutral position (and greater in flexion than in extension), while at C6 space was greatest during flexion. These findings were consistent with Muhle et al.s observation in regards to shortening of subarachnoid space during extension along with folding of ligamentum flavum, thereby reducing cord length.
Miura et al. [17] prospectively analyzed 20 patients with symptomatic CSM who underwent laminoplasty. They utilized the L-value, a measurement taken in the sagittal plane which describes the distance between the midline of the cord and the posterior aspect of the vertebral column. Measurements were taken pre- and postoperatively with dMRI. Regardless of surgery or position, L-values were smallest at C4-5. Postoperatively, the cord shifted dorsally in all positions, although the degree of cord motion was not related to surgical outcomes.

In a similar study, Miyata et al. [18] report changing L-values in 40 patients who underwent laminoplasty for symptomatic CSM. 20 of these patients had preoperative dMRI images which were used for controls, for a total of 60 scans. Postoperatively, the cord moved dorsally in all positions, supporting the findings of Miura et al. described above.

\section{Range of motion}

Fujimori et al. [11] compared postoperative range of motion (ROM) in patients with ossification of the posterior longitudinal ligament (OPLL) or CSM treated with laminoplasty. ROM in CSM patients significantly decreased from $32.7^{\circ}$ preoperatively to $24.4^{\circ}$ postoperatively, while ROM in OPLL patients decreased from $34.4^{\circ}$ to $20.8^{\circ}$. Loss of ROM was significantly greater in the OPLL group. In the CSM group, clinical outcomes were associated with changes in flexion and extension angles, but not overall ROM.

Machino et al. [15] reported changes in ROM and cervical alignment in 520 patients with symptomatic CSM who underwent laminoplasty. Mean cervical alignment was assessed by Cobb angles (C2-7), and was increased by $1.8^{\circ}$ (lordotic) postoperatively, while the mean total ROM was reduced by $6.6^{\circ}$.

\section{Disc changes}

After eliminating stenosis in the neutral position, Hayashi et al. [12] observed that many patients had undiagnosed stenosis during extension (8.3\%) and flexion (1.3\%). Further, disc bulge $>2.4 \mathrm{~mm}$, angular motion $>4.8^{\circ}$, and segmental kyphosis (C5-6) were correlated with an increased incidence of stenosis in extension, while disc bulge $>1.9$ $\mathrm{mm}$ predicted stenosis in a flexion MRI scan.

Fei et al. [10] utilized dMRI to assess translational and 
angular motion at levels adjacent to herniated discs in 407 patients with symptomatic disc herniations. They found that levels above the herniation experienced a $7.2 \%$ reduction in translational motion (per mm herniation) and no significant reduction in angular motion. Conversely, levels below the herniation experienced a $5.2 \%$ reduction in angular motion (per mm herniation) and no significant reduction in translational motion. Overall, there was no significant reduction in range of motion at levels above or below disc herniation as evaluated by $\mathrm{dMRI}$.

The position of the patient in the MRI also dictates the amount of compression on the disc. This is evidenced in biomechanical studies of lumbar spine where the compression of the disc is maximal at L3 when sitting and leaning forward. An MRI performed in this posture will definitely have a different disc height. Further, it is not uncommon patients do present with symptoms in certain upright positions which are relieved in lying down position. However, in cervical spine the the compression force is mainly exerted by the head and increases during upright position. dMRI performed in upright positions, add to this component to provide more realistic picture of the cervical spine.

\section{Outcomes and applications}

Segmental kyphosis is best diagnosed with dMRI, and is useful for preoperative planning, for example in deciding the number of levels to be decompressed [26,32]. Maruo et al. [16] noted that patients with $\mathrm{ROM}>20^{\circ}$ at $\mathrm{C} 2-7$ had worse outcomes after laminoplasty, and required instrumented fusion to prevent continued cord injury. This study implied that myelopathy often undercorrect without the utility of dynamic imaging.

Liu et al. correlated flexion/extension imaging with health-related quality of life scores (HRQOL), including the modified Japanese orthopedic association score, neck disability index, SF-36, and Nurick grade. They noted significant correlation between increased cervical lordosis in flexion and worse Nurick scoring. They hypothesized that such finding was due to the patient's attempt to minimize cord tension, compensate for deformity, and thereby decrease range of motion. Other observations include correlations between C7 translation over T1 and severity of symptoms, and sagittal slip of C2 or C4 and worse HRQOL measures. Reduced flexion and extension ROM correlated with worse SF-36 and Nurick scoring.
The applications for dMRI use have been gradually expanding. Seki et al. [23] demonstrated that patients with increased intramedullary signals during flexion had greater degree of neurological recovery, based on his evaluation and two-year follow-up of 121 patients with cervical myelopathy. The high intramedullary signal might indicate wide range of pathological changes which include edema, inflammation, and degeneration. Recently, dMRI has been used to evaluate Hirayama disease-juvenile muscular atrophy of the distal upper extremity (JMADUE)based on compression noted during flexion on dMRI in the presence of segmental spinal atrophy, detachment of the dorsal dura, and epidural venous congestion. The authors were able to refrain from operating on this genetic disorder, which stabilizes naturally with conservative intrventions [33-35]. This demonstrate the role of dynamic scan in diagnosing such disease with progressive weakness. Evans and Lollis [36] used dMRI to evaluate cord compression in a post-laminectomy case in which the paraspinal muscles were noted to compress the spinal cord during extension. This was managed by adding instrumented fusion with cross-links to maintain distance between the paraspinal muscles and the cord. dMRI studies in achondroplasia and rheumatoid arthritis patients have shown increased risk of dynamic compression at the cervico-medullary junction [37-39]. Dynamic imaging has also proven to be useful in children with skeletal dysplasia. Conditions like odontoid hypoplasia, os odontoideum, cerebrospinal fluid effacement, cord compression, spinal cord changes, and cervical canal narrowing have all been analyzed using dMRI [40].

\section{Conclusions}

Dynamic MRI is especially helpful in the evaluation of patients who have symptoms of CSM which are insufficient to warrant surgery. The neutral-position MRI does not reveal the severity of compression which is noted in dynamic images. This can, however, lead to the rapid deterioration of symptomatology often seen in elderly populations, due to disease progression and restricted, sedentary life style. Although segmental listhesis is detected in dynamic $\mathrm{X}$-ray films, mild listhesis is often overlooked. In these patients, dMRI will show the amount of cord impingement by soft tissues in addition to the bony spurs of spondylosis. dMRI is also helpful in patients with myelomalacia of the cord due to the repeated trauma of movement, which 
can paradoxically reveal adequate subarachnoid space in the neutral position.

We believe that dMRI has not yet been universally accepted for multiple reasons: undergoing the scan is a cumbersome and prolonged experience for patients already in significant distress from disease. There is often lack of coverage by patients' health insurance. Most importantly, there are no standardized measurements in dMRI to evaluate disease and dynamic change. For now, it can still be utilized by neurosurgeons in cases of discrepancy between neutral-position imaging and patient symptomatology.

\section{Conflict of Interest}

No potential conflict of interest relevant to this article was reported.

\section{References}

1. Kalsi-Ryan S, Karadimas SK, Fehlings MG. Cervical spondylotic myelopathy: the clinical phenomenon and the current pathobiology of an increasingly prevalent and devastating disorder. Neuroscientist 2013;19:409-21.

2. Klineberg E. Cervical spondylotic myelopathy: a review of the evidence. Orthop Clin North Am 2010;41:193-202.

3. Moore AP, Blumhardt LD. A prospective survey of the causes of non-traumatic spastic paraparesis and tetraparesis in 585 patients. Spinal Cord 1997;35:3617.

4. Toledano M, Bartleson JD. Cervical spondylotic myelopathy. Neurol Clin 2013;31:287-305.

5. Tracy JA, Bartleson JD. Cervical spondylotic myelopathy. Neurologist 2010;16:176-87.

6. Young WF. Cervical spondylotic myelopathy: a common cause of spinal cord dysfunction in older persons. Am Fam Physician 2000;62:1064-70.

7. Matsunaga S, Komiya S, Toyama Y. Risk factors for development of myelopathy in patients with cervical spondylotic cord compression. Eur Spine J 2015;24 Suppl 2:142-9.

8. Dalbayrak S, Yaman O, Firidin MN, Yilmaz T, Yilmaz M. The contribution of cervical dynamic magnetic resonance imaging to the surgical treatment of cervical spondylotic myelopathy. Turk Neurosurg 2015;25:36-42.
9. Endo K, Suzuki H, Nishimura H, Tanaka H, Shishido T, Yamamoto K. Kinematic analysis of the cervical cord and cervical canal by dynamic neck motion. Asian Spine J 2014;8:747-52.

10. Fei Z, Fan C, Ngo S, Xu J, Wang J. Dynamic evaluation of cervical disc herniation using kinetic MRI. J Clin Neurosci 2011;18:232-6.

11. Fujimori T, Le H, Ziewacz JE, Chou D, Mummaneni $\mathrm{PV}$. Is there a difference in range of motion, neck pain, and outcomes in patients with ossification of posterior longitudinal ligament versus those with cervical spondylosis, treated with plated laminoplasty? Neurosurg Focus 2013;35:E9.

12. Hayashi T, Wang JC, Suzuki A, et al. Risk factors for missed dynamic canal stenosis in the cervical spine. Spine (Phila Pa 1976) 2014;39:812-9.

13. Kim CH, Chung CK, Kim KJ, et al. Cervical extension magnetic resonance imaging in evaluating cervical spondylotic myelopathy. Acta Neurochir (Wien) 2014;156:259-66.

14. Liu S, Lafage R, Smith JS, et al. Impact of dynamic alignment, motion, and center of rotation on myelopathy grade and regional disability in cervical spondylotic myelopathy. J Neurosurg Spine 2015;23:690-700.

15. Machino M, Yukawa Y, Hida T, et al. Cervical alignment and range of motion after laminoplasty: radiographical data from more than 500 cases with cervical spondylotic myelopathy and a review of the literature. Spine (Phila Pa 1976) 2012;37:E1243-50.

16. Maruo K, Moriyama T, Tachibana T, et al. The impact of dynamic factors on surgical outcomes after double-door laminoplasty for ossification of the posterior longitudinal ligament of the cervical spine. J Neurosurg Spine 2014;21:938-43.

17. Miura J, Doita M, Miyata K, et al. Dynamic evaluation of the spinal cord in patients with cervical spondylotic myelopathy using a kinematic magnetic resonance imaging technique. J Spinal Disord Tech 2009;22:8-13.

18. Miyata K, Marui T, Miura J, et al. Kinetic analysis of the cervical spinal cord in patients after spinous process-splitting laminoplasty using a kinematic magnetic resonance imaging technique. Spine (Phila Pa 1976) 2006;31:E690-7.

19. Muhle C, Metzner J, Weinert D, et al. Classification system based on kinematic MR imaging in cervical spondylitic myelopathy. AJNR Am J Neuroradiol 
1998;19:1763-71.

20. Muhle C, Resnick D, Ahn JM, Sudmeyer M, Heller $M$. In vivo changes in the neuroforaminal size at flexion-extension and axial rotation of the cervical spine in healthy persons examined using kinematic magnetic resonance imaging. Spine (Phila Pa 1976) 2001;26:E287-93.

21. Muhle C, Weinert D, Falliner A, et al. Dynamic changes of the spinal canal in patients with cervical spondylosis at flexion and extension using magnetic resonance imaging. Invest Radiol 1998;33:444-9.

22. Muhle C, Wiskirchen J, Weinert D, et al. Biomechanical aspects of the subarachnoid space and cervical cord in healthy individuals examined with kinematic magnetic resonance imaging. Spine (Phila Pa 1976) 1998;23:556-67.

23. Seki S, Kawaguchi Y, Nakano M, et al. Clinical significance of high intramedullary signal on T2-weighted cervical flexion-extension magnetic resonance imaging in cervical myelopathy. J Orthop Sci 2015;20:9737.

24. White AP, Biswas D, Smart LR, Haims A, Grauer JN. Utility of flexion-extension radiographs in evaluating the degenerative cervical spine. Spine (Phila Pa 1976) 2007;32:975-9.

25. Zeitoun D, El Hajj F, Sariali E, Catonne Y, PascalMoussellard H. Evaluation of spinal cord compression and hyperintense intramedullary lesions on T2-weighted sequences in patients with cervical spondylotic myelopathy using flexion-extension MRI protocol. Spine J 2015;15:668-74.

26. Zhang L, Zeitoun D, Rangel A, Lazennec JY, Catonne Y, Pascal-Moussellard H. Preoperative evaluation of the cervical spondylotic myelopathy with flexionextension magnetic resonance imaging: about a prospective study of fifty patients. Spine (Phila Pa 1976) 2011;36:E1134-9.

27. Olsson SE. The dynamic factor in spinal cord compression; a study on dogs with special reference to cervical disc protrusions. J Neurosurg 1958;15:30821.

28. Tarlov IM, Klinger H, Vitale S. Spinal cord compression studies. I. Experimental techniques to produce acute and gradual compression. AMA Arch Neurol Psychiatry 1953;70:813-9.

29. Hattou L, Morandi X, Le Reste PJ, Guillin R, Riffaud L, Henaux PL. Dynamic cervical myelopathy in young adults. Eur Spine J 2014;23:1515-22.

30. Morishita Y, Maeda T, Ueta T, Naito M, Shiba K. Dynamic somatosensory evoked potentials to determine electrophysiological effects on the spinal cord during cervical spine extension: clinical article. J Neurosurg Spine 2013;19:288-92.

31. Wang MY, Widi G, Levi AD. The safety profile of lumbar spinal surgery in elderly patients 85 years and older. Neurosurg Focus 2015;39:E3.

32. Chen Y, Chen D, Wang X, et al. Significance of segmental instability in cervical ossification of the posterior longitudinal ligament and treated by a posterior hybrid technique. Arch Orthop Trauma Surg 2013;133:171-7.

33. Agundez M, Rouco I, Barcena J, Mateos B, Barredo J, Zarranz JJ. Hirayama disease: Is surgery an option? Neurologia 2015;30:502-9.

34. Arooj S, Mubarak F, Azeemuddin M, Sajjad Z, Jilani W. Hirayama disease, a rare cause of posture related cord compression: a case report from radiological perspective. J Pak Med Assoc 2013;63:1435-8.

35. Finsterer J, Loscher W, Wanschitz J, Baumann M, Quasthoff S, Grisold W. Hirayama disease in Austria. Joint Bone Spine 2013;80:503-7.

36. Evans LT, Lollis SS. Dynamic Compression of the Spinal Cord by Paraspinal Muscles following Cervical Laminectomy: Diagnosis Using Flexion-Extension MRI. Case Rep Radiol 2015;2015:275623.

37. Mukherjee D, Pressman BD, Krakow D, Rimoin DL, Danielpour M. Dynamic cervicomedullary cord compression and alterations in cerebrospinal fluid dynamics in children with achondroplasia: review of an 11-year surgical case series. J Neurosurg Pediatr 2014;14:238-44.

38. Iizuka H, lizuka Y, Kobayashi R, Nishinome M, Sorimachi $\mathrm{Y}$, Takagishi $\mathrm{K}$. The relationship between an intramedullary high signal intensity and the clinical outcome in atlanto-axial subluxation owing to rheumatoid arthritis. Spine J 2014;14:938-43.

39. Brouwer PA, Lubout CM, van Dijk JM, VleggeertLankamp CL. Cervical high-intensity intramedullary lesions in achondroplasia: aetiology, prevalence and clinical relevance. Eur Radiol 2012;22:2264-72.

40. Mackenzie WG, Dhawale AA, Demczko MM, et al. Flexion-extension cervical spine MRI in children with skeletal dysplasia: is it safe and effective? J Pediatr Orthop 2013;33:91-8. 